\title{
Resonant Tunneling through double-bended Graphene Nanoribbons
}

\author{
Z. Z. Zhang and Kai Chang* \\ SKLSM, Institute of Semiconductors, Chinese Academy of Sciences, P.O. Box 912, Beijing 100083, China \\ K. S. Chan \\ Department of Physics and Materials Science, City University of Hong Kong, Hong Kong, China
}

(Dated: October 30, 2018)

\begin{abstract}
We investigate theoretically resonant tunneling through double-bended graphene nanoribbon structures, i.e., armchair-edged graphene nanoribbons (AGNRs) in between two semi-infinite zigzag graphene nanoribbon (ZGNR) leads. Our numerical results demonstrate that the resonant tunneling can be tuned dramatically by the Fermi energy and the length and/or widths of the AGNR for both the metallic and semiconductor-like AGNRs. The structure can also be use to control the valley polarization of the tunneling currents and could be useful for potential application in valleytronics devices.
\end{abstract}

PACS numbers: 73.23.-b, 78.40.Gk, 73.40.Sx, 85.30.Mn

Graphene is a single layer of carbon atoms arranged in a hexagonal lattice. Recently, graphene samples have been fabricated experimentally by micro-mechanical cleavage of graphite [1]. This material has aroused the increasing attention due to its novel transport property that arises from its unique band structure: the conduction and valence bands meet conically at the two nonequivalent Dirac points, called $K$ and $K^{\prime}$ valleys, of the Brillouin zone, which show opposite chirality. Around the two points (called Diract points), the energy dispersion is linear and described by the massless Dirac equation. In graphene, the presence of edges can change the energy spectrum of the $\pi$-electron dramatically. Graphene nanoribbons (GNRs) have been fabricated by using conventional lithography and etching techniques [2, 3]. The electronic properties of a GNR depend very sensitively on the size and shape of edges, i.e., zigzag- and armchair-edged GNR. The zigzagedged graphene nanoribbons (ZGNRs) and armchairedged graphene nanoribbons (AGNRs) exhibit different band structures. For the ZGNRs, there are always the localized states appearing at the edge near the Dirac point. Therefore the ZGNRs exhibit metallic-like behavior. The AGNRs show metallic-like and semiconductorlike features alternatively as the width of nanoribbons increases [4]. Those features are very different from the conventional semiconductor quantum wire, provide possible ways to tailor the transport and optical properties of GNR [5], and pave a new path to potential applications of valleytronics device, e.g., the quantum point contact is used to realize a valley filter and a valley valve utilizing the edge state of ZGNRs [6]. Interestingly, the valleys $K$ and $K^{\prime}$ are decoupled for ZGNRs, but mixed for AGNRs [7]. It is natural to ask and image what happens when we construct a mesoscopic device by combining the ZGNRs and AGNRs.

\footnotetext{
*Electronic address: kchang@red.semi.ac.cn
}

In this work, we investigate theoretically the resonant tunneling through double-bended GNR structures, i.e., a AGNR in between two ZGNR leads (see Fig. 11). For transport through metallic-like AGNRs, electrons can not transmit perfectly but show a resonant tunneling behavior. For semiconductor-like AGNRs, the resonant tunneling is blocked when the Fermi energy $E_{F}$ is lower than the bandgap of AGNRs and displays similar resonant peaks when the Fermi energy $E_{F}$ exceeds the gap. Our theoretical results show that this kind of structure can control the valley polarization of tunneling currents.

The electronic states in graphene are described by a nearest-neighbor tight-binding Hamiltonian $H=$ $\sum_{<i, j>} t_{i j} c_{i}^{\dagger} c_{j}$, where $t_{i j}=-t(t=3.03 \mathrm{eV})$ is the transfer energy of the nearest-neighbour sites[11]. The conductance of the system is evaluated using the multichannel Landauer formula [8],

$$
G\left(E_{F}\right)=\frac{e^{2}}{\pi \hbar} \sum_{\mu} T_{\mu}\left(E_{F}\right), T_{\mu}\left(E_{F}\right)=\sum_{\nu}\left|t_{\mu, \nu}\left(E_{F}\right)\right|^{2},
$$

where $t_{\mu, \nu}\left(E_{F}\right)$ is a transmission coefficient from $\nu$-th channel in the left lead to $\mu$-th channel in the right lead at the Fermi energy $E_{F}$, calculated by a recursive Green's function method [9]. As schematically shown in Fig. 1, an AGNR is connected to two metallic semiinfinite ZGNR leads. The insets in Fig. 1 show the energy band structures of the left (right) ZGNR lead and middle AGNR. The Fermi energy $E_{F}$ can be tuned experimentally through the electric top- or back-gates [10]. In our calculation, all physical quantities are introduced dimensionlessly, e. g., the energy $E$ and the conductances are in units of $t$ and $e^{2} /(\pi \hbar)$, respectively.

First, we consider that the middle conductor of the double-bended GNRs consists of a metallic-like AGNR with $N_{C}=8$ and $\Delta_{M 1}=0.286$ (see the right inset of Fig.1). The left and right leads have the same width $N_{L}=N_{R}=20$ with $\Delta_{L 1}=0.113$ and $\Delta_{L 2}=0.189$ (see the left inset of Fig.1). Fig. 2 shows the contour 


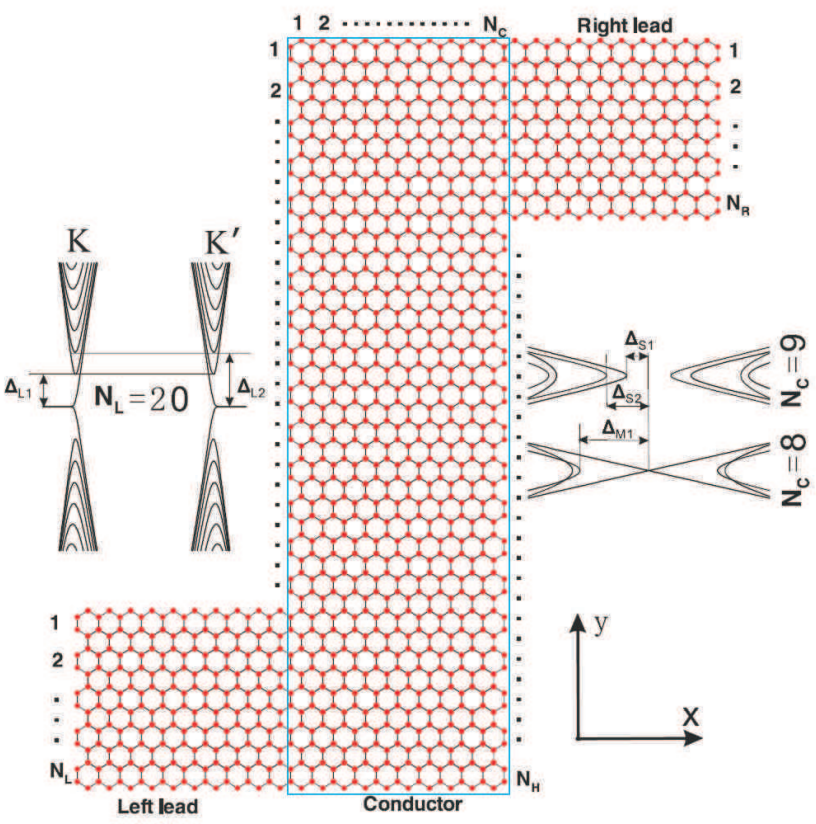

FIG. 1: (Color online) Schematic diagram of the proposed graphene nanoribbon structure. The left (right) inset describes the energy band structure for the left and right lead (AGR). The blue frame denotes the region of middle conductor, which consists of the AGNR with the length $N_{H}$ and width $N_{c}$.

plot of the conductance as functions of the length of the middle AGNR $\left(N_{H}\right)$ and the Fermi energy $\left(E_{F}\right)$. Fig. 2 demonstrates that there are three regimes indicated by the red triangles. The three regimes correspond to the opening of the second and third transverse modes. When $N_{H}=N_{L}=20$, the system will return to a perfect ZGNR and the conductance exhibits a step-like feature, i.e., $1,3,5 \cdots\left(e^{2} / \pi \hbar\right)$. When $N_{H}>N_{L}+N_{R}$, the resonant tunneling peaks appear regularly as the Fermi energy increases and become more and more as the length of AGNR region $N_{H}$ increases. This resonant tunneling behavior arises from the constructive interference effect when the electron wave propagates back and forth in the AGNR region. The incident electron can be completely reflected due to the destructive interference, especially when the energy of the incident electron is lower than the onset of the lowest mode of the left (right) lead, i.e., $E<\Delta_{L 1}$, though the middle AGNR is metallic with zero energy gap. For the fixed length, the resonance peaks broaden as the Fermi energy increase due to the enhanced coupling between the electron states in the AGNR and that in the ZGNR leads. When $\Delta_{L 1}<E<\Delta_{L 2}$, there are three propagating modes, two of which belong to the $K$ valley and one mode belongs to the $K^{\prime}$ valley. The similar oscillation can also be see when the incident energy is higher than the onset of the second modes in the left (right) lead.

Next, we consider the middle $\operatorname{AGNR}\left(N_{C}=9, \Delta_{S 1}=\right.$

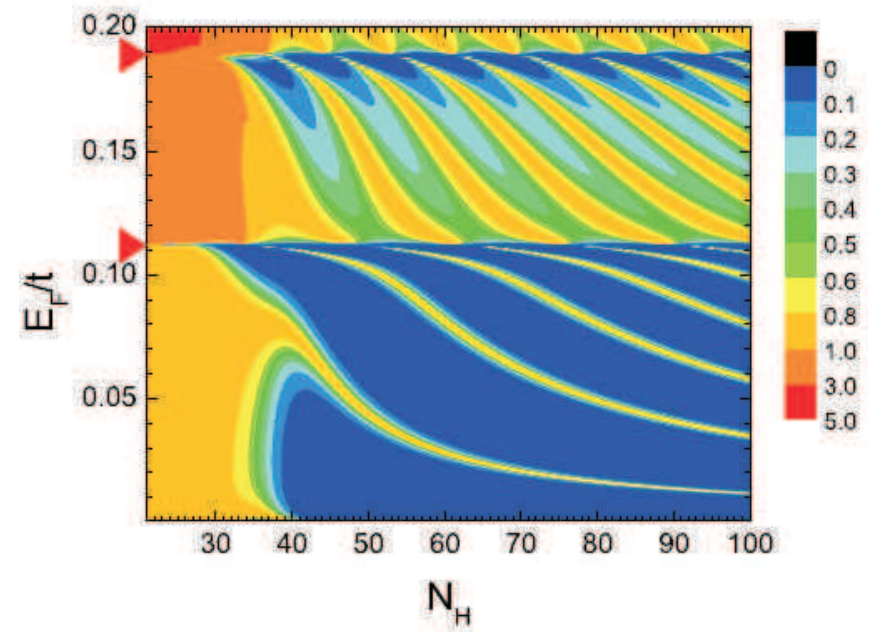

FIG. 2: (Color online) The contour plot of the conductance as a function of the length of the AGR $\left(N_{H}\right)$ and Fermi energy $\left(E_{F}\right) . \quad N_{L}=N_{R}=20, N_{A}=8$. The red triangles indicate the energies which corresponding the bottoms of the second and third transverse subbands in the left or right lead.

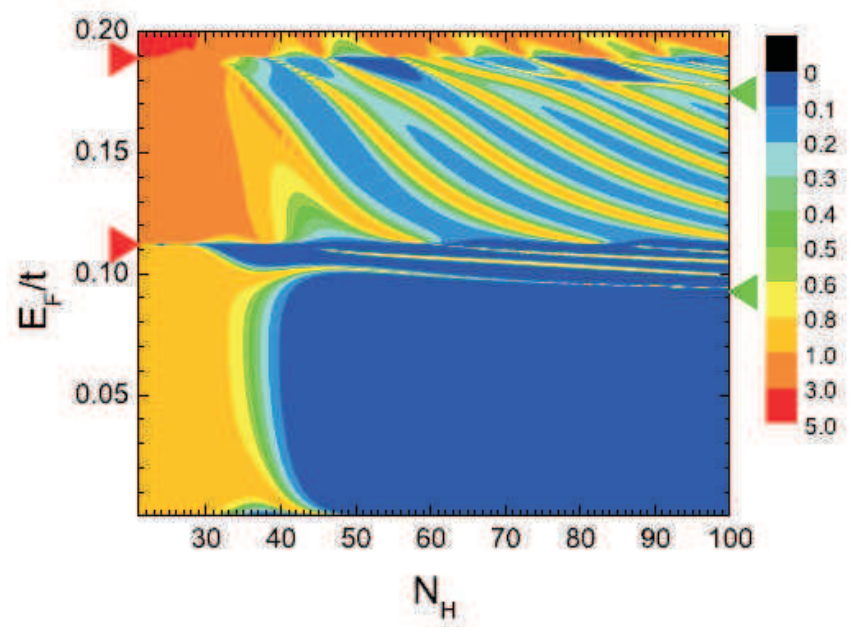

FIG. 3: (Color online) The same as Fig. 2 but for $N_{C}=$ 9 AGR conduct. The green triangles indicate the energies corresponding to $\Delta_{S 1}$ and $\Delta_{S 2}$

0.092 , and $\Delta_{S 2}=0.176$, see the right inset of Fig. 1) showing the semiconductor-like feature. When $N_{H}$ approaches to $N_{L}$, the situation is the same as for $N_{C}=8$ and show perfect transmission. But when $N_{H}$ increases and is larger than $N_{L}+N_{R}$, electron tunneling can be fully blocked for $0<E<\Delta_{S 1}$. This phenomena reveals that semiconductor AGNR behaves like an opaque barrier and can confine the electron between two AGNRs. For $\Delta_{S 1}<E<\Delta_{L 1}$ (see the left inset of Fig.1), similar resonance tunneling peaks appear, but are sharper than those through the metallic-like AGNR. When the incident energy increases further and is higher than $\Delta_{S 2}$, the resonant tunneling becomes more complicated, and 


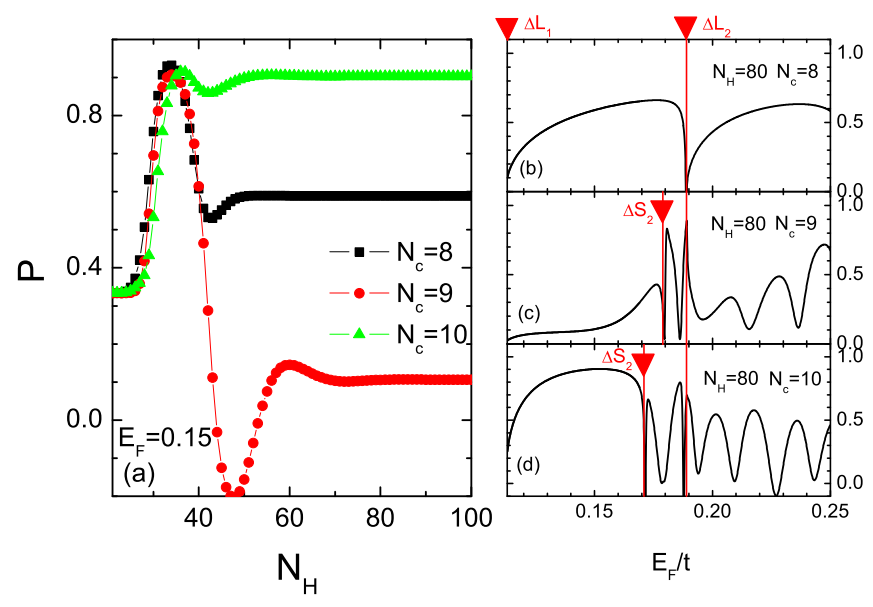

FIG. 4: (Color online) (a) Valley polarization (P) of the tunneling current as a function of the length $\left(N_{H}\right)$ of the middle conductor for the three different widths at the fixed chemical potential $E_{F}=0.15$. (b), (c), and (d) Valley polarization as a function of the chemical potential $(E)$ at the fixed length $\left(N_{H}=80\right)$ of middle AGNRs for $N_{C}=8,9$, and 10, respectively.

even shows crossing and anti-crossing features that are not found for the metallic AGNRs (see Fig. 2). The crossing and anticrossing behaviors are caused by the existence of the higher transverse modes of electron in the semiconductor-like AGNR region when the Fermi energy $E_{F}>\Delta_{S 2}$ (see the right inset of Fig.1). The absence of the crossing and anticrossing behavior for the metallic AGNR (see Fig. 2) arises from that the metallic AGNR only supports one channel when $E_{F}<\Delta_{M 1}$ (see the right inset of Fig.1).

Fig. 4(a) shows the valley polarization of the tunneling current as a function of the length of AGNR for the different widths $\left(N_{C}\right)$ of the AGNRs, where the valley polarization $P$ is defined as $P=\left(\sum_{\mu \in K} T_{\mu}-\sum_{\mu \in K^{\prime}} T_{\mu}\right) / \sum_{\mu} T_{\mu}$ [6]. There are the three channels propagating modes $\left(N_{m}=3\right)$ in the leads along the $x$ axis at $E_{F}=0.15$. Two channels belong to the $K$ valley and the other belongs to the $K^{\prime}$ valley. When the length of the AGNR approaches to the lead width, i.e., $N_{H}=N_{L}$, the structures become a perfect ZGNR and the valley polarization is equal to $1 / N_{m}\left(N_{m}=3\right)$ since there is no coupling between the $K$ and $K^{\prime}$ valleys. When $N_{H}>N_{L}$ (or $N_{R}$ ), the transmitted electron no longer belongs to the $K$ valley purely when the incident electron belonging to $K$ goes through AGNR due to the coupling between $K$ and $K^{\prime}$ valleys in the AGNR. As the length of the AGNR increases, the po- larization increases very fast and reaches the maximum at $N_{H} \approx 35$. When the length increases further, the valley polarizations decrease and saturate at specific values. The AGNRs with different widths have different saturated values. The AGNR with $N_{c}=3 n+1(n=3)$ show the highest saturated valley polarization, approaching to 0.9 . For the AGNRs with $N_{c}=3 n-1$, the saturated value is the lower. The saturated polarization of the AGNR with $N_{c}=3 n$ is the lowest and approaches to 0.1. Figs. 4(b), (c), and (d) show the valley polarizations as a function of the Fermi energy for different widths of AGNRs. When there is a single channel in the middle AGNR region, i.e., $E_{F}<\Delta_{M 1}$, for the metallic-like AGNR and $E_{F}<\Delta_{S 2}$ for the semiconductor-like AGNR, the valley polarization varies smoothly. Once the Fermi energy exceeds the critical energies $\left(\Delta_{M 1}\right.$ or $\left.\Delta_{S 2}\right)$, the valley polarization oscillates heavily (see Figs. 4(c) and (d)) because of the interference between different channels in the AGNR region and ZGNR leads which are opened orderly with increasing the Fermi energy. But the valley polarization in Fig. 4(b) still changes smoothly as the Fermi energy increases. This is because the AGNR only supports a single channel when the energy of the second channel is higher than the Fermi energy, i.e., $\Delta_{M 1}>E_{F}$ (see the right inset of Fig. 1). For the single incident channel case, i.e., $0<E_{F}<\Delta_{L 1}$, the valley polarization of tunneling current is always equal to 1 since there is a single channel belonging to the $K$ valley. From Fig. 4, one can see that the valley polarization of the tunneling current can be changed dramatically by tuning the Fermi energy and the length of the AGNR.

In summary, we have investigated theoretically resonant tunneling through a double-bended GNR, i.e., an AGNR in between two ZGNR leads. Our numerical results demonstrate that the resonant tunneling can be tuned dramatically by the Fermi energy and the length and/or widths of the AGNR for both the metallic and semiconductor-like AGNRs. The valley polarization saturate as the length of the AGNRs increases, and the saturated valley polarizations depend sensitively on the widths of the AGNRs. The structure we proposed can be used to manipulate the valley polarization of the tunneling current and should be useful for potential application in valleytronics devices.

\section{Acknowledgments}

This work is supported by the NSF of China Grant No. 60525405 .
[1] K. S. Novoselov, A. K. Geim, S. V. Morozov, D. Jiang, Y. Zhang, S. V. Dubonos, I. V. Grigorieva, and A. A. Firsov, Science 306, 666 (2004).

[2] Z. H. Chen, Y. M. Lin, M. J. Rooks, and P. Avouris,
Physica E 40, 228 (2007).

[3] M. Y. Han, B. Ozyilmaz, Y. B. Zhang, and P. Kim, Phys. Rev. Lett. 98, 206805 (2007).

[4] K. Nakada, M. Fujita, G. Dresselhaus, and M. S. Dres- 
selhaus, Phys. Rev. B 54, 17954 (1996).

[5] K. Wakabayashi, Phys. Rev. B 64, 125428 (2001).

[6] A. Rycerz, J. Tworzydlo, and C. W. J. Beenakker, Nature Physics 3, 172 (2007).

[7] L. Brey and H. A. Fertig, Phys. Rev. B 73, 235411 (2006).

[8] M. Büttiker, Y. Imry, R. Landauer, and S. Pinhas, Phys. Rev. B 31, 6207 (1985).
[9] T. Ando, Phys. Rev. B, 44, 8017 (1991)

[10] B. Ozyilmaz, P. Jarillo-Herrero, D. Efetov, D. A. Abanin, L. S. Levitov, and P. Kim, Phys. Rev. Lett. 99 (2007).

[11] R. Saito, G. Dresselhaus, and M. S. Dresselhaus, Physical Properties of Carbon Nanotubes (Imperial College Press, London, 1998). 